\title{
Impacto da COVID-19 na gravidade e prognóstico de indivíduos com obesidade: uma revisão sistemática
}

\author{
Impact of COVID-19 on the gravity and \\ prognosis of individuals with obesity: a \\ systematic review
}

\section{Tamires Alexandrina de Araújo' 1 (1) Renata Ferreira de Moura ${ }^{2}$ (1) Nathielly Oliveira de Almeida ${ }^{3}$ (1)}

\author{
'Autora para correspondência. Centro Universitário Dom Pedro II (Salvador). Bahia, Brasil. tami.alexandrina@outlook.com \\ 2Universidade Salvador (Feira de Santana). Bahia, Brasil. renataferreirademoura@outlook.com.br \\ ${ }^{3}$ Faculdade de Ciências e Empreendedorismo (Santo Antônio de Jesus). Bahia, Brasil. nathiellyoliveiradealmeida@gmail.com
}

\begin{abstract}
RESUMO | INTRODUÇÃo: A maioria dos pacientes com obesidade apresenta resistência à insulina e hiperatividade do sistema renina-angiotensina-aldosterona, o que pode estar relacionado aos piores resultados perante infecção pelo SARS-CoV 2. Além disso, a enzima conversora de angiotensina 2 tem maior expressão no tecido adiposo quando comparado com o pulmão, sendo suscetível a invasão do vírus nos adipócitos tornando-o um importante reservatório viral permitindo propagação para demais órgãos. OBJETIVO: Identificar se a obesidade pode ser preditor de maior gravidade e pior prognóstico da Doença do Coronavírus 2019 (COVID-19). MATERIAIS E MÉTODOS: Revisão sistemática sob o código PROSPERO CRD42020200617 com estudos observacionais através das bases de dados PubMed, Portal Regional da BVS, SciELO, Science Direct e Cochrane, e buscas manuais por meio do cruzamento "Prognosis" OR "Patient Acuity" AND "Coronavirus Infections" AND "Obesity". Foram incluídos estudos observacionais que avaliaram o impacto da COVID-19 em indivíduos com obesidade de ambos os sexos que apresentaram pontuação $\geq 7$ na escala Newcastle-Ottawa. RESULTADOS: Foram incluídos 9 estudos totalizando uma amostra de 179.047 pacientes adultos com idade entre 18 a 80 anos, com IMC mínimo $<24 \mathrm{~kg} / \mathrm{m} 2$ e máximo $>35 \mathrm{~kg} / \mathrm{m} 2$. Verificou-se que indivíduos com obesidade apresentam aumento das taxas de admissão de cuidados agudos e críticos, necessidade ventilação mecânica invasiva (VMI), pneumonia e desenvolvem COVID-19 grave, aumentando assim seu tempo de permanência hospitalar. CONCLUSÃO: Indivíduos com obesidade desenvolvem maior gravidade e pior prognóstico da COVID-19, visto que apresentam aumento das taxas de admissão de cuidados agudos e críticos, necessidade de VMI, tempo de permanência hospitalar, gravidade e letalidade.
\end{abstract}

DESCRITORES: Prognóstico. Gravidade do paciente. Infecções por coronavírus. Obesidade.
ABSTRACT | INTRODUCTION: A marked proportion of patients with obesity has insulin resistance and hyperactivity of the reninangiotensin-aldosterone system, which may be related to the worse results caused by SARS-CoV 2 . In addition, the angiotensin 2-converting enzyme has greater expression in adipose tissue when compared to the lung, being susceptible to the entry of the virus in the adipocytes making it an important viral reservoir allowing the spread to too many organs. OBJECTIVE: To identify whether obesity can be a more serious predictor and worse prognosis for Coronavirus Disease 2019 (COVID-19). MATERIALS AND METHODS: Systematic review under the code CRD42020200617 with observational studies through the PubMed databases, the VHL Regional Portal, SciELO, Science Direct and Cochrane, and manual searches using the "Prognosis" OR "Patient Acuity" AND " Coronavirus Infections " AND " Obesity ". Observational studies that assess the impact of COVID-19 in common with obesity of both sexes that assess a score $\geq 7$ on the Newcastle-Ottawa scale were included. RESULTS: 9 studies were included, totaling a sample of 179,047 adult patients aged 18 to 80 years, with a minimum $\mathrm{BMI}<24$ $\mathrm{kg} / \mathrm{m} 2$ and a maximum $>35 \mathrm{~kg} / \mathrm{m} 2$. It was found that with obesity, he wishes to present an increase in the admission rates for acute and mandatory care, invasive mechanical necessity IMV), pneumonia and develop severe COVID-19, thus increasing their hospital stay. CONCLUSION: Individuals with obesity develop greater severity and worse prognosis for COVID-19, since there is an increase in admission rates for acute and mandatory care, need for IMV, length of hospital stay, severity and lethality.

DESCRIPTORS: Prognosis. Patient acuity. Coronavirus infections. Obesity. 


\section{Introdução}

A atual pandemia da síndrome respiratória aguda grave do coronavírus (SARS-CoV-2), comumente conhecida como Doença do Coronavírus 2019 (COVID-19), coloca-nos frente a um cenário com sérias repercussões para os sistemas de saúde no Brasil e no mundo. Essa infecção gera uma grave insuficiência respiratória hipoxêmica, consequentemente resultando em superlotação das unidades de terapia intensiva (UTI), carência de equipamentos e profissionais, e números de mortos significativos, principalmente na população com condições crônicas de saúde prévias .

O SARS-CoV-2 tem capacidade de adentrar nas células humanas por meio de ligação direta com os receptores da enzima conversora de angiotensina 2 (ECA 2) na superfície celular ${ }^{2-3}$. Subsequentemente promove desajuste da expressão dessa enzima tornando-a incapaz de cumprir seus efeitos protetores nos órgãos ${ }^{2-3}$. Sabe-se que uma parte considerável dos pacientes obesos apresenta resistência à insulina e hiperatividade do sistema renina-angiotensina-aldosterona, e esse fato pode estar relacionado aos piores resultados perante infecção por este vírus². Evidências têm demonstrado que a COVID-19 pode piorar a resistência à insulina em pessoas com Diabetes Mellitus Tipo 2 e Tipo 1, principalmente aqueles com obesidade. Mesmo na forma mais leve, a COVID-19 pode induzir um meio pró-inflamatório, que pode ser responsável por uma redução da sensibilidade à insulina ${ }^{4}$.

Mesmo sabendo que a ECA 2 pertence ao sistema renina angiotensina aldosterona ${ }^{5}$, ainda não foi demonstrado que pessoas com obesidade possuem alguma alteração (quantidade e/ou qualidade) dessa enzima. Há uma hipótese de que a maior expressividade da ECA 2 no tecido adiposo de indivíduos com obesidade pode aumentar a infecção pelo SARSCoV-2 e a receptividade a esse tecido 5,6 . A ECA 2 pode permitir a entrada do vírus nos adipócitos tornando-o um importante reservatório viral permitindo propagação para demais órgãos².

Visto que a obesidade é um problema de saúde pública, responsável pelo aumento da incidência de hipertensão, diabetes e demais comorbidades ${ }^{7}$, nosso estudo apresenta como relevância clínica maior esclarecimento da relação do SARS-CoV-2 e a obesidade, permitindo um melhor direcionamento de abordagens para esses pacientes. Portanto, a presente revisão sistemática tem como objetivo identificar se a obesidade pode ser preditor de maior gravidade e pior prognóstico da COVID-19.

\section{Métodos}

Revisão sistemática realizada conforme as recomendações do PRISMA (Preferred reporting items for systematic reviews and meta-analyses $)^{8}$, registrada na PROSPERO (International prospective register of systematic reviews) sob o código PROSPERO CRD42020200617, para responder à pergunta através da estratégia PECO: indivíduos com obesidade apresentam maior gravidade e consequentemente pior prognóstico na COVID-19?

Foram incluídos estudos observacionais que avaliaram o impacto da COVID-19 em indivíduos com obesidade, de ambos os sexos, que apresentaram pontuação $\geq 7$ na escala Newcastle-Ottawa ${ }^{9}$. Em contrapartida, foram excluídos estudos com indivíduos acometidos por outro tipo de corona-vírus ou demais vírus respiratórios, bem como estudos com dados incompletos e revisões de literatura.

As buscas dos artigos científicos foram realizadas no mês de julho 2020 nas plataformas virtuais de dados PubMed, Portal Regional da BVS, SciELO, Science Direct e Cochrane Library por dois autores independentes, juntamente com busca manual, com as seguintes combinações dos Descritores em Ciência da Saúde (DECS) e do Medical Subject Headings (MeSH): "Prognosis" OR "Patient Acuity" AND "Coronavirus Infections" AND "Obesity". Uma nova busca foi realizada no mês de setembro de 2020 para possíveis atualizações de novos estudos.

A avaliação da qualidade dos estudos incluídos foi realizada pela escala Newcastle-Ottawa, sua mensuração foi calculada em três componentes: seleção dos grupos (zero a quatro pontos); comparabilidade (zero a dois pontos); e exposição/resultado (zero a três pontos). Essa mensuração foi apresentada numericamente, sendo que cada asterisco (*) correspondeu a um ponto, totalizando de zero a nove pontos.

O processo de triagem se deu inicialmente com a realização de leitura dos artigos a partir dos títulos e resumos, gerando assim a primeira exclusão, logo após a leitura dos manuscritos, ocorreu à segunda exclusão, e em seguida a extração dos desfechos de 
interesse, que incluem o desenho do estudo, características das amostras, o grupo controle, os desfechos analisados e principais resultados obtidos que foram exibidos em tabela, com apresentação dos valores e intervalo de confiança ou valor " $p$ " de significância, sendo considerado pelos estudos significância quando $p<0,05$.

\section{Resultados}

Das 98 referências encontradas através das estratégias de busca, apenas 3 foram excluídas por duplicidade. Do restante, 79 foram excluídas após leitura dos títulos e resumos. Por fim, 9 estudos foram incluídos para análise considerando os critérios de elegibilidade (Figura 1).

Figura 1. Fluxograma da triagem e seleção dos artigos que investigaram a relação da COVID-19 em indivíduos com obesidade no período de julho a setembro 2020

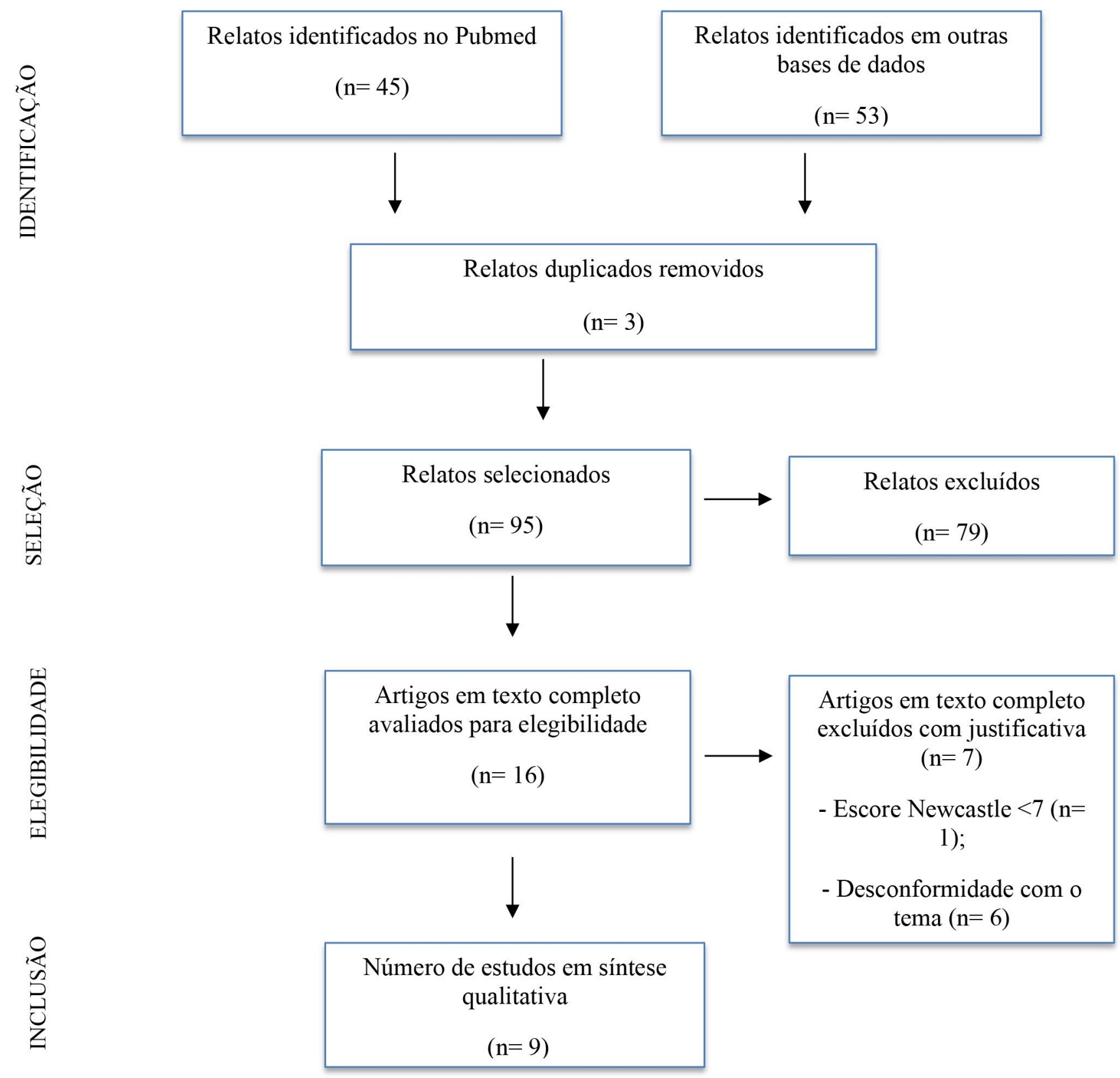


Os estudos incluídos foram conduzidos no México, China, Estados Unidos e França ${ }^{10-18}$. O tempo de seguimento dos estudos variou de 1 a 2 meses, sendo cinco artigos ${ }^{11-13,16,18}$ com 1 mês de seguimento. Todos eram coortes retrospectivos observacionais, exceto dois que eram coorte prospectiva, e incluíram um total de 179.047 pacientes adultos, de 18 a 80 anos de idade, em sua maioria do sexo masculino, com escores variados de gravidade e IMC, sendo considerado $>30 \mathrm{~kg} / \mathrm{m} 2$, segundo a Organização Mundial da Saúde e $>25 \mathrm{~kg} / \mathrm{m} 2$ considerando o ponto de corte para populações asiáticas ${ }^{19}$.

A qualidade metodológica dos estudos incluídos foi alta, (Tabela1). Os escores variaram de 7 a 9 pontos. Metanálises não puderem ser conduzidas devido ao desenho metodológico predominante dos estudos incluídos nesta revisão ser coorte observacional.

Tabela 1. Avaliação de qualidade dos artigos selecionados de acordo com a escala Newcastle-Ottawa

\begin{tabular}{|c|c|c|c|c|c|c|}
\hline \multirow[t]{2}{*}{ AUTOR } & \multirow{2}{*}{$\begin{array}{l}\text { TIPO } \\
\text { ESTUDOS }\end{array}$} & \multirow[t]{2}{*}{ DE } & \multicolumn{3}{|c|}{ ITENS AVALIADOS } & \multirow[t]{2}{*}{ TOTAL } \\
\hline & & & SELEÇÃO & COMPARABILIDADE & EXPOSIÇÃO & \\
\hline $\begin{array}{l}\text { BELLO-CHAVOLLA et } \\
\text { al. }^{10}\end{array}$ & Coorte & & $\star \star \star * *$ & $\star *$ & $* \star *$ & 9 \\
\hline CAl et al. ${ }^{11}$ & Coorte & & $\star * \star *$ & $\star *$ & $* *$ & 8 \\
\hline GAO et al. ${ }^{12}$ & Coorte & & $* * *$ & $\star *$ & ** & 7 \\
\hline $\begin{array}{l}\text { HAJIFATHALIAN et } \\
\text { al. }^{13}\end{array}$ & Coorte & & $* * * *$ & ** & ** & 8 \\
\hline HU et al. ${ }^{14}$ & Coorte & & 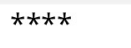 & * & ** & 7 \\
\hline KALLIGEROS et al. ${ }^{15}$ & Coorte & & $\star * \star *$ & $\star *$ & $* \star *$ & 9 \\
\hline LIGTHER et al. ${ }^{16}$ & Coorte & & $\star * \star *$ & * & ** & 7 \\
\hline SIMONNET et al. ${ }^{17}$ & Coorte & & $\star * \star *$ & $\star *$ & $* \star *$ & 9 \\
\hline ZENG et al. ${ }^{18}$ & Coorte & & $* * *$ & ** & ** & 7 \\
\hline
\end{tabular}

Avaliação da qualidade dos estudos incluídos pela escala Newcastle-Ottawa, sendo que cada quesito atendido corresponde a um asterisco.

Abaixo se encontram descritos os resultados encontrados após extração de dados dos estudos incluídos. 


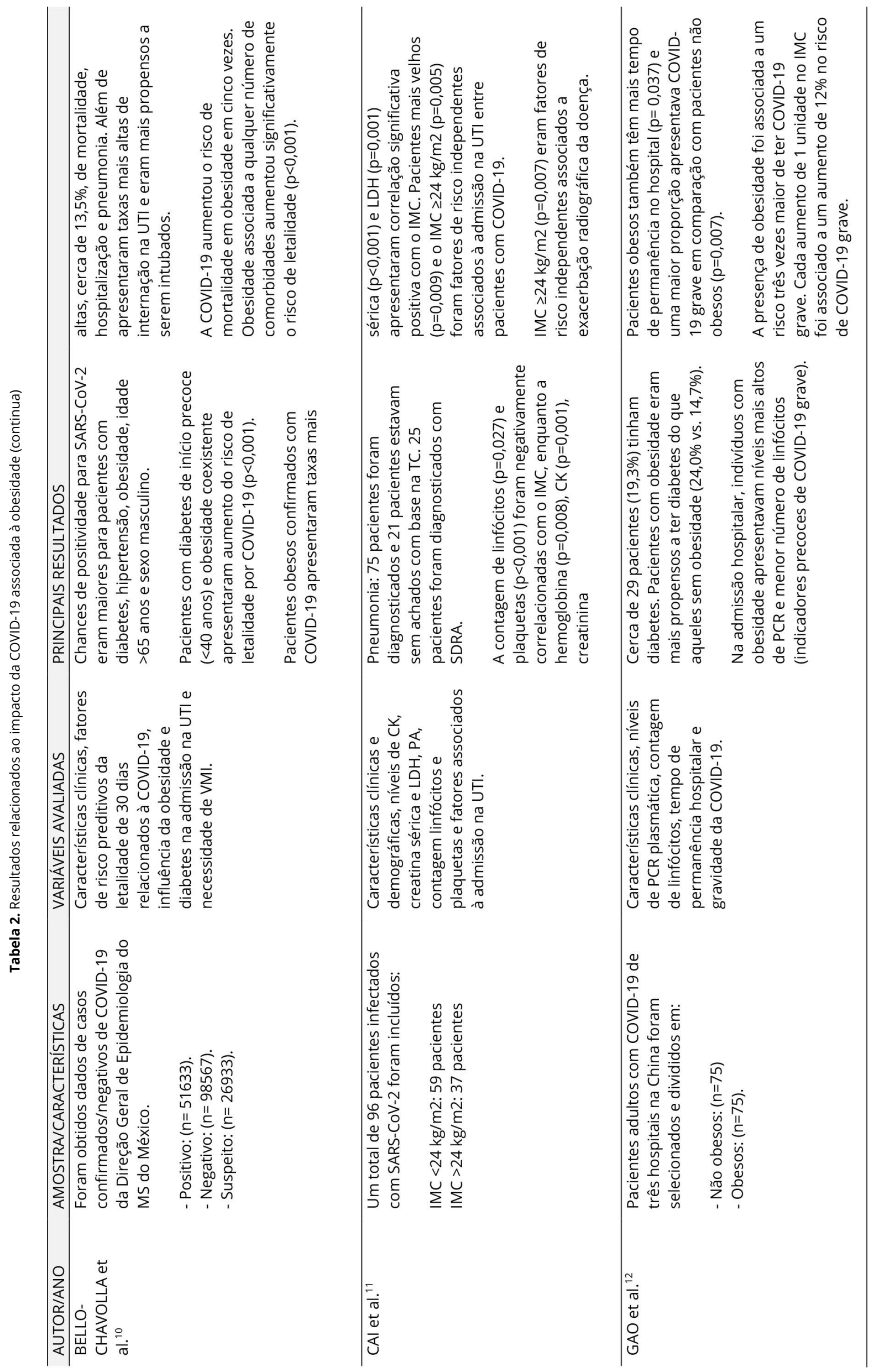




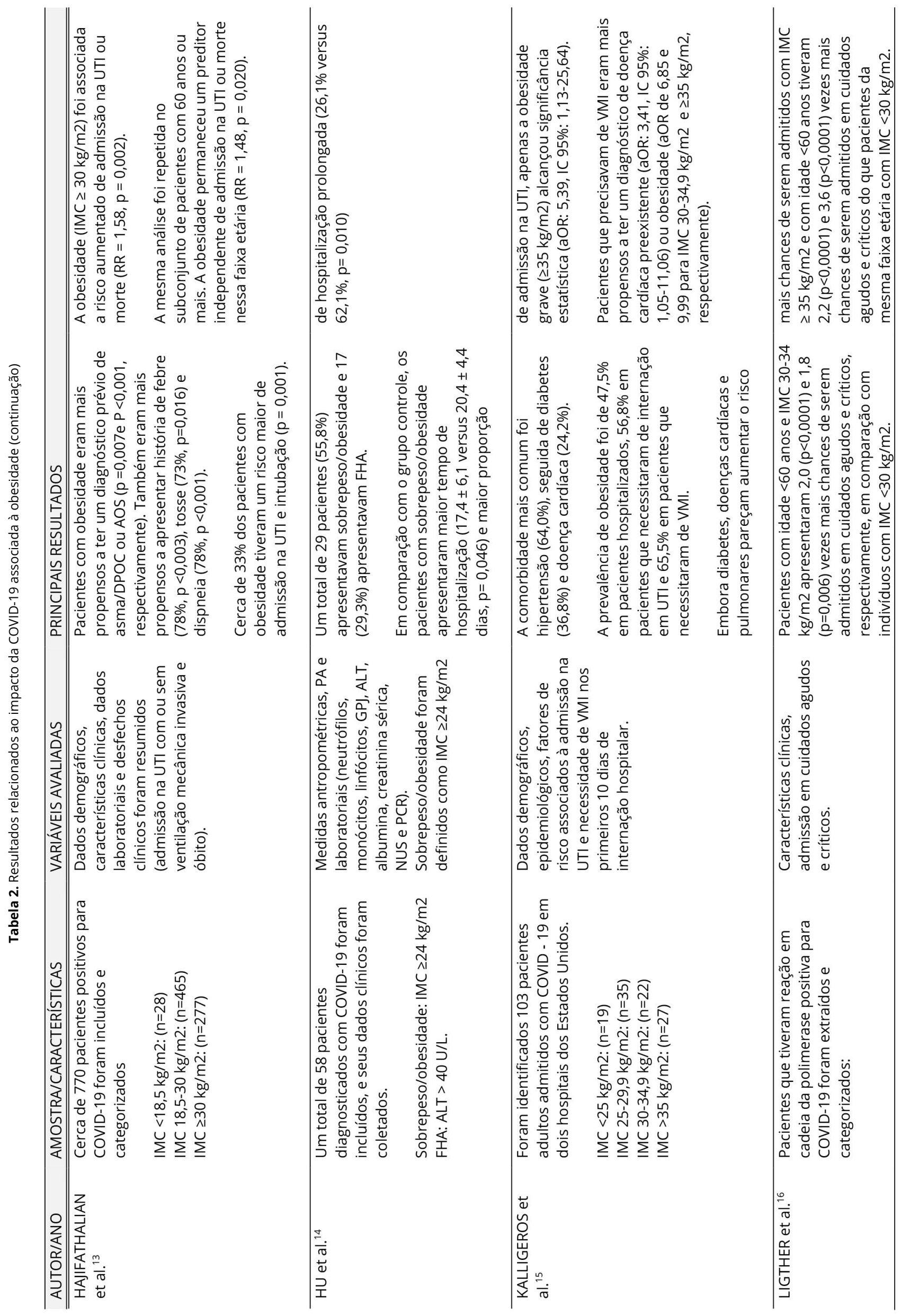




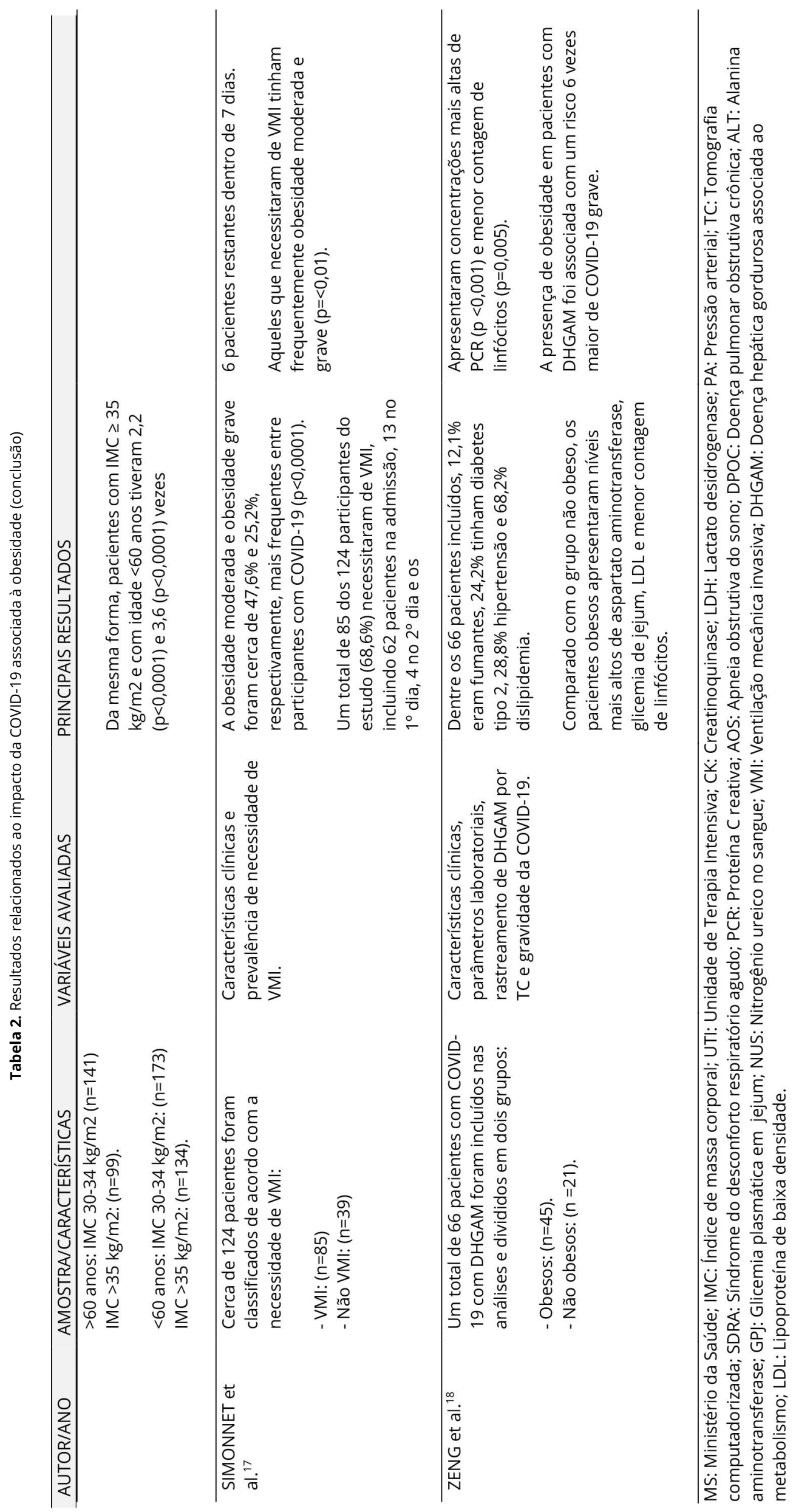




\section{Discussão}

Foi possível observar que pacientes com obesidade apresentam maior taxa de hospitalização, de cuidados agudos e admissão na UTI, pneumonia, síndrome do desconforto respiratório agudo e VMI (ventilação mecânica invasiva) quando comparados com indivíduos não obesos ${ }^{10,11,13-17}$. Esses pacientes apresentam uma sobrecarga da musculatura respiratória, sendo três vezes maior, de modo que o consumo total de oxigênio respiratório é elevado em $14 \%$. Uma infecção viral aguda pode ocasionar desarmonias adicionais entre a capacidade de geração de força da musculatura respiratória e as demandas geradas à respiração, acarretando um aumento do risco de insuficiência respiratória $^{20}$. Verificou-se uma maior permanência hospitalar nesse público, a qual pode ser justificada pelo fato de que pacientes com obesidade apresentam maior predisposição em apresentar COVID-19 mais grave ${ }^{12}$, nos casos de obesos com DHGAM esse risco chega a ser seis vezes maior ${ }^{18}$. Além disso, foi observado que indivíduos com obesidade e doença cardíaca preexistente são mais propensos a necessidade de VMI.

Em relação à letalidade, indivíduos com obesidade apresentaram risco cinco vezes maior, quando comparados aos não obesos, essa condição associada à comorbidades, independentemente da quantidade, aumentou os riscos significativamente. $O$ diabetes de início precoce ( $<40$ anos de idade) associada à obesidade coexistente ressaltou compatibilidade com maiores taxas de mortalidade em indivíduos com COVID-19. Esse achado reflete a noção de que o diabetes tipo 2 de início precoce pode gerar um aumento do risco de mortalidade em pacientes mais jovens ${ }^{10}$.

O IMC apresentou relação com maiores taxas tanto de admissão na UTI, quanto na necessidade VMI e na exacerbação radiográfica da COVID-1911,13-17. Além disso, a obesidade demonstrou associação com maior gravidade da doença, visto que cada unidade de IMC aumenta em $12 \%$ os riscos de COVID-19 grave $^{12}$, da mesma forma que a obesidade $>35 \mathrm{~kg} / \mathrm{m} 2$ é capaz de aumentar os riscos de admissão na UTI卢. A produção desproporcional de adipocinas (especialmente leptina e adiponectina) tem chamado atenção, em virtude de que atuam como moléculas sinalizadoras com amplos efeitos em diversos sistemas, incluindo os pulmões, a qual pode fornecer uma explicação mecanicista relacionada ao perigo da associação da obesidade com a COVID-19 grave. $O$ tecido adiposo também tem a capacidade de produzir um maior número de outras adipocinas, incluindo resistina e visfatina, que atingem o sistema imunológico e podem estar associadas aos desfechos adversos da sepse ${ }^{21}$.

A idade avançada também se mostrou um fator de risco independente associado à admissão na UTI entre os pacientes com COVID-1911. Em um outro estudo foi identificado que indivíduos com obesidade com IMC 30$34 \mathrm{~kg} / \mathrm{m} 2$ ou maior que $35 \mathrm{~kg} / \mathrm{m} 2$, com idade inferior a 60 anos, tiveram maiores chances de serem admitidos em cuidados agudos e críticos em relação aos pacientes da mesma faixa etária com IMC $>30 \mathrm{~kg} / \mathrm{m} 2^{16}$. Em contrapartida, outras evidências demonstram que a associação da obesidade com admissão na UTI é verdadeira em todas as faixas etárias, bem como no subconjunto de pacientes com mais de 60 anos, especificamente ${ }^{13}$.

Referente aos parâmetros laboratoriais, como contagem de linfócitos ${ }^{11,12,18}$ e de plaquetas foi visto que estes estavam negativamente correlacionados ao IMC, enquanto a contagem de hemoglobina, $\mathrm{CK}$, creatinina sérica e LDH apresentaram correlação significativamente positiva ${ }^{12}$. Além disso, indivíduos obesos apresentam níveis mais altos de PCR, aspartato aminotransferase, glicemia em jejum e LDL ${ }^{12,18}$. Tanto os níveis elevados de PCR quanto menores números de linfócitos são considerados indicadores precoces da COVID-19 grave, confirmando assim a vulnerabilidade destes indivíduos a uma maior gravidade e pior prognóstico para a doença em questão ${ }^{18}$.

É importante salientar que alguns dos estudos incluídos nesta revisão apresentaram a obesidade associada a outras comorbidades $10,12,13,15,18$, sobretudo a diabetes, o que pode acarretar um maior risco para o acometimento da forma mais grave pela COVID-19. Dentre as limitações encontradas para elaboração dessa revisão estão o número reduzido de estudos que avaliassem a gravidade e prognóstico de indivíduos com obesidade com diagnóstico da COVID-19, além da escassez da avaliação de variáveis pulmonares. Outra limitação seria que poucos estudos avaliaram a associação da obesidade com outras comorbidades, é plausível que esse fato possa resultar em um acometimento mais grave da COVID-19 e consequentemente um pior prognóstico.

Ainda são necessários estudos que investiguem a repercussão da obesidade como um fator para uma maior gravidade e pior prognóstico da COVID-19 ao longo prazo. Entretanto, nosso estudo contribui de forma significativa para a prática clínica, pois evidencia que 
a COVID-19 associada à obesidade gera maior tempo de permanência hospitalar, necessidade de VMI, bem como maiores taxas de mortalidade. Esses resultados são importantes e permitem um melhor direcionamento de abordagens para esses pacientes.

\section{Conclusão}

Os indivíduos com obesidade desenvolvem maior gravidade e pior prognóstico da COVID-19, visto que apresentam aumento das taxas de admissão de cuidados agudos e críticos, necessidade de VMI, maior tempo de permanência hospitalar, gravidade e letalidade, além de apresentarem alteração nos parâmetros laboratoriais como linfócitos, plaquetas e hemoglobina, CK, creatinina sérica, LDH, PCR, aspartato aminotransferase, glicemia em jejum e LDL. Diante disso, são necessárias realizações de novos estudos para maiores evidências e melhor direcionamento de abordagens para esses indivíduos.

\section{Agradecimento}

À Professora Carole Cavalcante da Conceição Aguiar pelo apoio e incentivo durante a redação do artigo científico.

\section{Contribuições dos autores}

Araújo TA, Moura RF e Almeida NO participaram da concepção, delineamento, busca e análise dos dados da pesquisa, interpretação dos resultados, redação do artigo científico, aprovação da versão final.

\section{Conflitos de interesses}

Nenhum conflito financeiro, legal ou político envolvendo terceiros (governo, empresas e fundações privadas, etc.) foi declarado para nenhum aspecto do trabalho submetido (incluindo, mas não se limitando a subvenções e financiamentos, participação em conselho consultivo, desenho de estudo, preparação de manuscrito, análise estatística, etc.).

\section{Referências}

1. Pinto TF, Carvalho CRF. SARS CoV-2 (COVID-19): lessons to be learned by Brazilian Physical Therapists. Brazilian Journal of Physical Therapy. 2020;24(3):185-186. doi: 10.1016/j.

bjpt.2020.04.004
2. Sanchis-Gomar F, Lavie CJ, Mhera MR, Henry BM, Lippi G. Obesity and Outcomes in COVID-19: When an Epidemic and Pandemic Collide. Mayo Clin Proc. 2020;95(7):1445-1453. doi: 10.1016/j.mayocp.2020.05.006

3. Vaduganathan M, Vardeny O, Michel T, McMurray JJV, Pfeffer MA, Solomon SD. Renin-Angiotensin-Aldosterone System Inhibitors in Patients with Covid-19. N Engl J Med. 2020;382(17):1653-1659. doi: 10.1056/NEJMsr2005760

4. Pal R, Bhadada SK. COVID-19 and diabetes mellitus: An unholy interaction of two pandemics. Diabetes Metab Syndr. 2020;14(4):513-517. doi: 10.1016/j.dsx.2020.04.049

5. Patel VB, Zhong JC, Grant MB, Oudit GY. Role of the ACE2/ Angiotensin 1-7 Axis of the Renin-Angiotensin System in Heart Failure. Circ Res. 2016;118(8):1313-26. doi: $10.1161 /$ CIRCRESAHA. 116.307708

6. Pasquarelli-do-Nascimento G, Braz-de-Melo HA, Faria SS, Santos IO, Kobinger GP, Magalhães KG. Hypercoagulopathy and Adipose Tissue Exacerbated Inflammation May Explain Higher Mortality in COVID-19 Patients With Obesity. Front Endocrinol (Lausanne). 2020;11:530. doi: $10.3389 /$ fendo.2020.00530

7. Jiang SZ, Lu W, Zong XF, Ruan HY, Liu Y. Obesity and hypertension. Exp Ther Med. 2016;12(4):2395-2399. doi: 10.3892/ etm.2016.3667

8. Moher D, Liberati A, Tetzlaff J, Altman DG, PRISMA Group. Preferred reporting items for systematic reviews and metaanalyses: the PRISMA statement. PLoSMed 2009;6(7):e1000097. doi: 10.1371/journal.pmed.1000097

9. Stang A. Critical evaluation of the Newcastle-Ottawa scale for the assessment of the quality of nonrandomized studies in metaanalyses. Eur J Epidemiol. 2010;25(9):603-5. doi: 10.1007/s10654010-9491-z

10. Bello-Chavolla OY, Bahena-López JP, González-Díaz A, Márquez-Salinas A, Aguilar-Salinas CA, Naveja J et al. Predicting mortality due to SARS-CoV-2: A mechanistic score relating obesity and diabetes to COVID-19 outcomes in Mexic. MedRxiv. 2020;105(8):1-28. doi: 10.1101/2020.04.20.20072223

11. Cai SH, Liao W, Chen SW, Liu LL, Liu SY, Zeng ZD. Association between obesity and clinical prognosis in patients infected with SARS-CoV-2. Infectious Diseases of Poverty. 2020;9(1):1-7. doi: 10.1186/s40249-020-00703-5

12. Gao F, Zeng KI, Wang XB, Sun QF, Pan KH, Wang TY et al. Obesity Is a Risk Factor for Greater COVID-19 Severity. Diabetes Care. 2020;43(8):1-3. doi: 10.2337/dc20-0682

13. Hajifathalian K, Kumar S, Newberry C, Shah S, Fortune B, Krisko T et al. Obesity is Associated with Worse Outcomes in COVID-19: Analysis of Early Data from New York City. Obesity. 2020;28(9):1606-1612. doi: 10.1002/oby.22923 
14. Hu X, Pan X, Zhou W, Gu X, Shen F, Yang B et al. Clinical epidemiological analyses of overweight/obesity and abnormal liver function contributing to prolonged hospitalization in patients infected with COVID-19. Int J Obes. 2020;44:1784-1789. doi: 10.1038/s41366-020-0634-3

15. Kalligeros M, Shehadeh F, Mylona EK, Benitez G, Beckwith CG, Chan PA et al. Association of Obesity with Disease Severity Among Patients with Coronavirus Disease 2019. Obesity (Silver Spring). 2020;28(7):1200-1204. doi: 10.1002/oby.22859

16. Lighter J, Phillips M, Hochamn S, Sterling S, Johnson D, Francois F et al. Obesity in Patients Younger Than 60 Years Is a Risk Factor for COVID-19 Hospital Admission. J Pediatric Infect Dis Soc. 2020;71(15):896-897. doi: 10.1093/cid/ciaa415

17. Simonnet A, Chetboun M, Poissy J, Raverdy V, Noulette J, Duhamel $A$ et al. High Prevalence of Obesity in Severe Acute Respiratory Syndrome Coronavirus-2 (SARS-CoV-2) Requiring Invasive Mechanical Ventilation. Obesity. 2020;28(7):1195-1199. doi: 10.1002/oby.22831
18. Zeng Kl, Gao F, Wang X, Sun Q, Pan K, Wang T et al. Obesity as a risk factor for greater severity of COVID-19 in patients with metabolic associated fatty liver disease. Metabolism. 2020;108:154244. doi: 10.1016/j.metabol.2020.154244

19. WHO Expert Consultation. Appropriate body-mass index for Asian populations and its implications for policy and intervention strategies. Lancet. 2004;363(9403):157-63. doi: 10.1016/S01406736(03)15268-3

20. Severin R, Arena R, Lavie CJ, Bond S, Phillips AS. Respiratory Muscle Performance Screening for Infectious Disease Management Following COVID-19: A Highly Pressurized Situation. Am J Med. 2020;133(9):1025-1032. doi: 10.1016/j. amjmed.2020.04.003

21. Post A, Bakker SJL, Dullaart RPF. Obesity, adipokines and COVID-19. Eur J Clin Invest. 2020;50:e13313. doi: 10.1111/ eci.13313 